\title{
Should Clinicians Start Measuring Flow Mediated Dilation Response in Patients with Systemic Lupus Erythematosus?
}

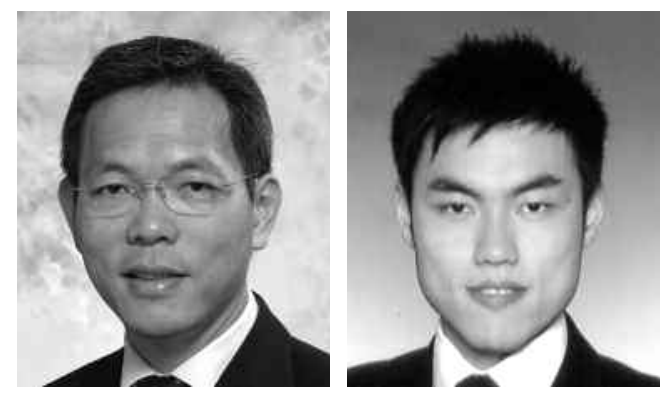

Premature atherosclerosis as a major cause of late mortality in systemic lupus erythematosus (SLE) was first confirmed by Urowitz and his colleagues in the $1970 \mathrm{~s}^{1}$. The authors described a bimodal pattern of mortality based on a cohort of 81 patients followed for 5 years. Of the 5 patients who died late in the course of SLE, all had a myocardial infarction either close to or at the time of death. Subsequently, many studies have confirmed patients with SLE are exposed to an increased risk of cardiovascular (CV) disease $e^{2,3,4}$. Prevention of atherosclerosis development is an important goal in the longterm management of patients with SLE. In general, these patients are more exposed to traditional CV risk factors. For example, a higher prevalence of metabolic syndrome has been demonstrated in SLE patients than in controls $^{5,6}$. Regular surveillance and prompt treatment of $\mathrm{CV}$ risk factors may thus improve longterm survival. In addition, there is evidence that repeated systemic inflammation plays a pivotal role in the development of CV complications in $\mathrm{SLE}^{7}$. Previous studies have shown patients with worse disease with frequent inflammatory flares are at a greater risk of developing $\mathrm{CV}$ disease in the long term ${ }^{8,9}$. Clearly, therefore, maintaining the patient's disease in remission is another important preventive strategy against CV complications in SLE.

Additionally, ability to predict CV disease will go a long way to improve the longterm prognosis of SLE. A number of surrogate markers have been developed to detect preclinical atherosclerosis. These include Doppler ultrasound (US) measurement of carotid intima media thickness ${ }^{10}$, detection of left ventricular abnormalities ${ }^{11}$, and multidetector computerized tomographic scanning to measure the extent of coronary calcification ${ }^{12}$. While the performance of these tools is generally good, the changes found are often those of established atherosclerosis. Thus, a surrogate marker that predates atherosclerosis development will be useful. Such a marker should also relate well to the pathogenic mechanisms of CV disease in SLE; be noninvasive, objective, and easy to interpret; and allow repeated measurement so clinicians can monitor the effectiveness of interventions. The endothelium, an important organ that maintains thrombosis and hemostasis, is believed to be the link between repeated inflammatory insults and the onset of atherosclerosis. Inflammation causes endothelial damage and functional abnormalities ${ }^{13,14}$. Endothelial damage and functional changes have been shown to be among the first events in the development of atherosclerosis, and correlate strongly with the prevalence of $\mathrm{CV}$ events ${ }^{15}$. It is possible to demonstrate endothelial damage through the measurement of various endothelium released products such as nitric oxide (NO), prostacyclin, plasminogen activator inhibitor, von Willebrand factor antigen, and adhesion molecules. Unfortunately, however, these substances tend to be nonspecific and do not correlate well with endothelial functional changes and subsequent development of $\mathrm{CV}$ disease.

Recently, 2 methods - flow mediated dilation (FMD) and pulse wave analysis - have been widely used as measurements of endothelial reactivity. Both have been validated and shown to be predictors of CV disease ${ }^{16}$. FMD uses US to view the brachial artery and measure transient changes in its diameter in response to shear stress as a result of antegrade blood flow blockade following blood pressure cuff occlusion. This produces vasodilation of the resistance arteries distal to where the blood flow is blocked. On deflation of the cuff, a reactive hyperemia occurs in the brachial artery, causing dilation. FMD is measured as the percentage change in brachial artery diameter in response to the increased blood flow. This vasodilation is dependent on endothelial NO, which is produced as a consequence of shear stress on the endothelium. The results are then compared with those obtained after the administration of an exogenous nitrate, e.g., sublingual glyceryl trinitrate, an endothelial independent vasodilation. This technique is relatively easy to master and is noninvasive and objective. Abnormal FMD has been found in SLE patients with CV

See ED but not EI FMD is reduced in SLE patients without vascular events, page 1296

Personal non-commercial use only. The Journal of Rheumatology Copyright @ $\odot 2011$. All rights reserved. 
disease ${ }^{17,18}$. So, should clinicians start measuring FMD in these patients as a marker to predict and monitor the development of $\mathrm{CV}$ disease and its subsequent progression? FMD measurement may also help us better understand the relationship between disease flares, antiinflammatory therapies, and endothelial dysfunction. Before we embark on regular FMD measurements in patients with SLE, it is important to delineate the clinical relevance of the 2 FMD measurements, endothelium-dependent (ED) and endothelium-independent (EI) FMD. This was the primary objective of Mak and his colleagues' metaanalysis study on ED-FMD and EI-FMD in patients with SLE reported in this issue of The Journal ${ }^{19}$.

In Mak, et al's study ${ }^{19}$, the authors performed a systematic review of case-controlled studies that compared ED-FMD and/or EI-FMD between vascular event-naive SLE patients and matched healthy subjects. Using a metaanalysis, the authors assessed whether ED-FMD and EI-FMD were impaired in patients with SLE without a history of vascular event, and explored factors that might moderate impaired FMD in these patients. ED-FMD was found to be significantly lower in SLE patients than healthy subjects, while no differences in EI-FMD were found between the 2 groups of subjects. The mixed-model metaregression analysis was then performed to evaluate if demographic and clinical factors affected the differences in ED-FMD patients and controls. Interestingly, age and duration of SLE at the time of FMD measurement significantly narrowed the difference of ED-FMD between the 2 subject groups, while gender, smoking status, menopause, diabetes mellitus, body mass index, blood pressure, fasting lipid profile, C-reactive protein, and the use of prednisolone did not.

The results reported by Mak and colleagues are relevant and interesting. First, that only ED-FMD, which is dependent on endothelial NO release, was found to be different between SLE patients and control subjects is in accord with the proposed pathoetiologic role of endothelial damage and dysfunction in atherosclerosis. Second, ED-FMD measurement is convenient and noninvasive, and may be repeated regularly to monitor endothelial functional changes during the lupus disease course and response to treatment of the underlying disease, as well as traditional CV risk factors.

Some precautions are needed, however, when advocating the regular measurement of ED-FMD in patients with SLE. While it is relatively easy to master the skills of this test, the technical aspects of measuring brachial reactivity are not standard, as suggested by a systematic review by Bots, et $a l^{20}$. The same method should be used during repeated measurements and direct comparison of results should only be made if there are no variations in the examination technique. Further, results should be interpreted with caution in patients with increasing age ( $>55$ years) and advanced disease. It is not immediately apparent why age narrows the gap between SLE and control ED-FMD. A possible explanation is that endothelial dysfunction due to disease in SLE patients reaches a ceiling after a certain age and cannot worsen as much as controls with aging or disease duration. Whatever the explanation, ED-FMD may not be a useful marker to identify or monitor endothelial damage, and hence atherosclerosis development, in lupus patients with advanced age and long disease duration, according to Mak and colleagues.

Most previous efforts of the scientific community in the management of SLE have focused on controlling the underlying immune aberrations and secondary inflammatory changes. We now know that premature atherosclerosis, which is probably the consequence of harmful effects of inflammation on the endothelium, is a significant cause of late deaths in these patients. To be able to predict and monitor the progress of CV disease in SLE will go a long way in improving the longterm outcome of this condition. ED-FMD measurement is reliable, noninvasive, easy to use, reproducible, and pathogenically relevant for this purpose. Clinicians should start measuring ED-FMD prospectively as part of their management program for patients with SLE.

CHAK SING LAU, MBChB, MD, (Hons) FRCP, Professor and Chief,

TOMMY CHEUNG, MBBS, MRCP(UK), FHKAM,

Specialist Registrar,

Division of Rheumatology and Clinical Immunology,

Li Ka Shing Faculty of Medicine,

The University of Hong Kong,

Hong Kong, China

Address correspondence to Dr. Lau; E-mail: cslau@hku.hk

\section{REFERENCES}

1. Urowitz MB, Bookman AA, Koehler BE, Gordon DA, Smythe HA, Ogryzlo MA. The bimodal mortality pattern of systemic lupus erythematosus. Am J Med 1976;60:221-5.

2. Manzi S, Meilahn EN, Rairie JE, Conte CG, Medsger TA Jr, Jansen-McWilliams L, et al. Age-specific incidence rates of myocardial infarction and angina in women with systemic lupus erythematosus: comparison with the Framingham Study. Am J Epidemiol 1997;145:408-15.

3. Nikpour M, Urowitz MB, Gladman DA. Premature atherosclerosis in systemic lupus erythematosus. Rheum Dis Clin N Am 2005; 31:329-58.

4. Petri M. Clinical research in systemic lupus erythematosus: immediate relevance to clinic practice. Int J Rheum Dis 2011;14:1-5.

5. Chung CP, Avalos I, Oeser A, Gebretsadik T, Shintani A, Raggi P, et al. High prevalence of the metabolic syndrome in patients with systemic lupus erythematosus: association with disease characteristics and cardiovascular risk factors. Ann Rheum Dis 2007;66:208-14.

6. Sabio J, Zamora-Pasadas M, Jiménez-Jáimez J, Albadalejo F, Vargas-Hitos J, Rodríguez del Aguila MD, et al. Metabolic syndrome in patients with systemic lupus erythematosus from Southern Spain. Lupus 2008;17:849-59.

7. Szekanecz Z, Koch AE. Vascular involvement in rheumatic diseases: 'vascular rheumatology'. Arthritis Res Ther 2008;10:224.

8. Roman MJ, Shanker BA, Davis A, Lockshin MD, Sammaritano L, Simantov R, et al. Prevalence and correlates of accelerated atherosclerosis in systemic lupus erythematosus. N Engl J Med 2003;349:2399-406.

9. Asanuma Y, Oeser A, Shintani AK, Turner E, Olsen N, Fazio S, et al.

Personal non-commercial use only. The Journal of Rheumatology Copyright @ 2011 . All rights reserved. 
Premature coronary-artery atherosclerosis in systemic lupus erythematosus. N Engl J Med 2003;349:2407-15.

10. O'Leary DH, Polak JF, Kronmal RA, Manolio TA, Burke GL, Wolfson SK Jr, et al. Carotid-artery intima and media thickness as a risk factor for myocardial infarction and stroke in older adults. N Engl J Med 1999;340:14-22.

11. Yan RT, Bluemke D, Gomes A, Burke G, Shea S, Liu K, et al. Regional left ventricular myocardial dysfunction as a predictor of incident cardiovascular events MESA (Multi-Ethnic Study of Atherosclerosis). J Am Coll Cardiol 2011;57:1735-44.

12. Greenland P, Bonow RO, Brundage BH, Budoff MJ, Eisenberg MJ, Grundy SM, et al. American College of Cardiology Foundation Clinical Expert Consensus Task Force (ACCF/AHA Writing Committee to Update the 2000 Expert Consensus Document on Electron Beam Computed Tomography); Society of Atherosclerosis Imaging and Prevention; Society of Cardiovascular Computed Tomography. J Am Coll Cardiol 2007;49:378-402.

13. Gonzalez MA, Selwyn AP. Endothelial function, inflammation, and prognosis in cardiovascular disease. Am J Med 2003;115 Suppl 8A:99(S)-106(S)

14. Devaraj S, Yun JM, Adamson G, Galvez J, Jialal I. C-reactive protein impairs the endothelial glycocalyx resulting in endothelial dysfunction. Cardiovasc Res 2009;84:479-84.

15. Le Brocq M, Leslie SJ, Milliken P, Megson IL. Endothelial dysfunction: from molecular mechanisms to measurement, clinical implications, and therapeutic opportunities. Antioxid Redox Signal 2008;10:1631-74.
16. Celermajer DS, Sorensen KE, Bull C, Robinson J, Deanfield JE. Endothelium-dependent dilation in the systemic arteries of asymptomatic subjects relates to coronary risk factors and their interaction. J Am Coll Cardiol 1994;24:1468-74.

17. Lima DS, Sato EI, Lima VC, Miranda F Jr, Hatta FH. Brachial endothelial function is impaired in patients with systemic lupus erythematosus. J Rheumatol 2002;29:292-7.

18. Johnson SR, Harvey PJ, Floras JS, Iwanochko M, Ibanez D, Gladman DD, et al. Impaired brachial artery endothelium dependent flow mediated dilation in systemic lupus erythematosus: preliminary observations. Lupus 2004;13:590-3.

19. Mak A, Liu Y, Ho RC-M. Endothelium-dependent but not endothelium-independent flow-mediated dilation is significantly reduced in patients with systemic lupus erythematosus without vascular events: a metaanalysis and metaregression. J Rheumatol 2011;38:1296-303.

20. Bots ML, Westerink J, Rabelink TJ, de Koning EJ. Assessment of flow-mediated vasodilation (FMD) of the brachial artery: effects of technical aspects of the FMD measurement on the FMD response. Eur Heart J 2005;26:363-8.

J Rheumatol 2011;38:1231-3; doi:10.3899/jrheum.110635 